\title{
Enterococcus Faecalis Infective Endocarditis and Colorectal Carcinoma: Case of New Association Gaining Ground
}

\author{
Zubair Khan ${ }^{a, c}$, Nauman Siddiqui ${ }^{b}$, Muhammad Wasif Saif ${ }^{b}$
}

\begin{abstract}
Mostly Streptococcus bovis (S. bovis) bacteremia and endocarditis $(60 \%)$ has been found to be associated with underlying colorectal cancer (CRC). Enterococcus faecalis (E. faecalis) bacteremia and endocarditis has no identifiable source in most of the cases. $E$. faecalis is part of normal gut flora that can translocate through the intestine and cause the systemic infection. With any intestinal lesion or tumor, the barrier is breached and the gut flora like E. faecalis can translocate and cause infection. A 55-years-old male known to have non-ischemic cardiomyopathy with implantation of automated implantable cardioverter defibrillator (AICD) and atrial fibrillation presented with weight loss, fever and back pain. He was diagnosed to have E. faecalis bacteremia and subsequent endocarditis and osteomyelitis of T7 - T8 and L4 - L5 vertebrae. He underwent colonoscopy for screening of malignancy because of his age and presenting symptoms suggestive of one. The colonoscopy found pedunculated polyp in sigmoid colon, and after biopsy the histology revealed an invasive well differentiated mucinous adenocarcinoma, with focal squamous differentiation. He underwent removal of AICD and antibiotic treatment for infective endocarditis and osteomyelitis. He underwent sigmoid colectomy with pathology of removed specimen showing adenocarcinoma with negative margins and lymph nodes. In many of the patients with E. faecalis endocarditis, if identifiable the source is genitourinary tract. But in most of the cases the source of E. faecalis bacteremia is unidentified. There is some evidence to suggest that in patients with unidentified source, colonoscopy may reveal a hidden early stage $\mathrm{CRC}$ or adenoma. We conclude that in cases of E. faecalis bacteremia and endocarditis with unidentified source, colonoscopy should be considered if feasible to rule out the diagnosis of CRC.
\end{abstract}

Keywords: Infective endocarditis; Colorectal cancer; E. faecalis bacteremia; E. faecalis infective endocarditis

Manuscript submitted February 28, 2018, accepted March 26, 2018

aDepartment of Internal Medicine, University of Toledo Medical Center, Toledo, OH, USA

bDepartment of Hematology and Oncology, Tufts Medical Center, Boston, MA, USA

${ }^{\mathrm{c}}$ Corresponding Author: Zubair Khan, Department of Internal Medicine, University of Toledo, 3000 Arlington Avenue, Toledo, $\mathrm{OH}$ 43614, USA.

Email: zubair.khan@utoledo.edu

doi: https://doi.org/10.14740/gr996w

\section{Introduction}

There is a strong evidence of association between Streptococcus gallolyticus, formerly Streptococcus bovis (S. bovis) bacteremia and colorectal cancer (CRC) [1]. The prevalence of CRC in patients with $S$. Bovis undergoing colonoscopy is approximately $60 \%,[2]$ and both American [3] and European [4] guidelines recommend systematic colonoscopy in these patients. Enterococcus faecalis (E. faecalis) formerly classified as part of the group D Streptococcus system is a Grampositive, commensal bacterium inhabiting the gastrointestinal tracts of humans and other mammals. Enterococci are becoming one of the most common causes of infection in elderly population [5]. E. faecalis is the most common among enterococci responsible for $63-81 \%$ of cases [6], and malignancy is the most common comorbidity [7]. E. faecalis endocarditis is the third leading cause of infective endocarditis, being responsible for $5 \%$ to $15 \%$ of cases. The source of bacteremia in majority of these cases is undetermined and if identifiable, it is mostly genitourinary tract. There has been some evidence in favor of association between enterococcal endocarditis and hidden CRC [8]. Here we report a case of E. faecalis endocarditis associated with CRC.

\section{Case Report}

A 55-year-old gentleman known to have non-ischemic cardiomyopathy with automated implantable cardioverter defibrillator (AICD) implantation and atrial fibrillation presented with the complaint of low grade fever, productive cough, back pain, fatigue and weight loss. His labs revealed normocytic anemia $(\mathrm{Hb} 11.4 \mathrm{~g} / \mathrm{dL}$ ), the hepatitis C antibody (anti-HCV) positive, blood cultures positive for E. faecalis. Chest X-ray showed right lower lobe pneumonia. Trans-esophageal echocardiography for workup of bacteremia revealed aortic valve vegetation. His computerized tomography (CT) abdomen and pelvis was negative. His social history was positive for being active smoker and 20 pack years of smoking history. He was treated for pneumonia with oral ciprofloxacin at the dose of $500 \mathrm{mg}$ PO q12h for 14 days. Further workup done for the back pain revealed osteomyelitis of T7 - T8 vertebrae and L4 - L5 vertebrae.

His history of unintentional weight loss of $30 \mathrm{lb}$ in 2 months with normocytic anemia prompted the workup for hid- 


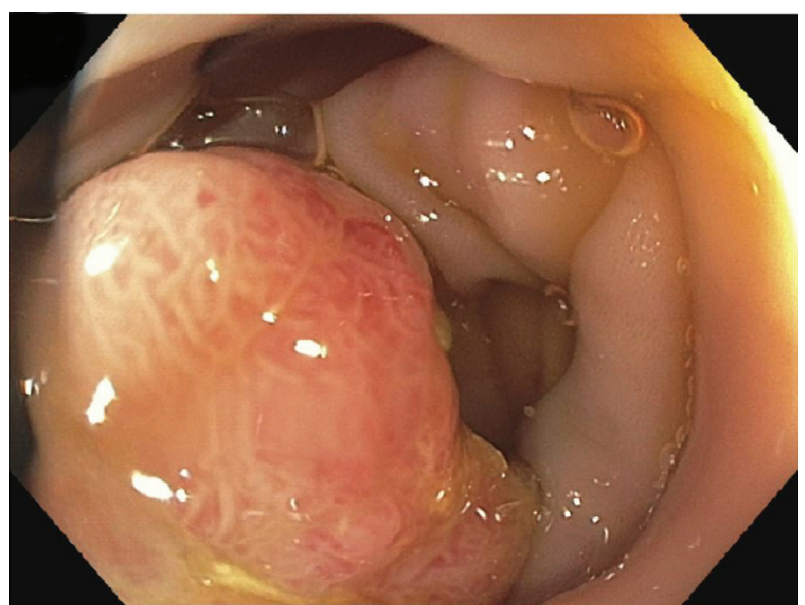

Figure 1. Large pedunculated polyp.

den malignancy. His AICD was also removed because of the high suspicion of seeding during bacteremia. He subsequently underwent colonoscopy that showed the large $2 \mathrm{~cm} \times 2 \mathrm{~cm}$ pedunculated polyp with $5 \mathrm{~cm}$ stalk found in sigmoid colon at $30 \mathrm{~cm}$ (Fig. 1). The pathology showed well differentiated invasive mucinous adenocarcinoma, with focal squamous differentiation (Fig. 2). The colorectal surgery team did a left hemicolectomy. The pathology from that surgery showed minimal residual adenocarcinoma, no metastasis in 18 retrieved lymph nodes $(0 / 18)$, and negative margins with tumor classification of T1N0M0. He was treated for endocarditis and osteomyelitis meanwhile with IV antibiotics and started treatment for $\mathrm{HCV}$ after surgery for stage $1 \mathrm{CRC}$. The stage $1 \mathrm{CRC}$ was thus the source of $E$. faecalis bacteremia and the resultant complications of endocarditis and osteomyelitis.

\section{Discussion}

Enterococci are Gram-positive, catalase-negative, facultative anaerobes that are seen under direct microscopy as diplococci in short chains. E. faecalis is part of normal gut flora and is responsible for human disease via translocation from gut wall and leading to systemic infection [9].

$\mathrm{CRC}$ is the third most common cancer in men and women in the United States of America. The most common etiology is sporadic, with genetic and environmental factors playing an important role. Other established risk factors include male gender, older age, inflammatory bowel disease and positive family history. The US Preventive Services Task Force (USPSTF) currently recommends routine screening for colorectal cancer between the ages of 50 and 75 using fecal occult blood testing, sigmoidoscopy or colonoscopy.

The relationship between $S$. bovis infective endocarditis and CRC is well established [10]; the association between $E$. faecalis endocarditis and CRC is yet to be established. Some data even suggest that the E. faecalis may be responsible for mutagenesis of colonic cells and resultant malignancy and intestinal lesions that then lead to translocation of E. faecalis into blood stream [11]. The most frequent identifiable source

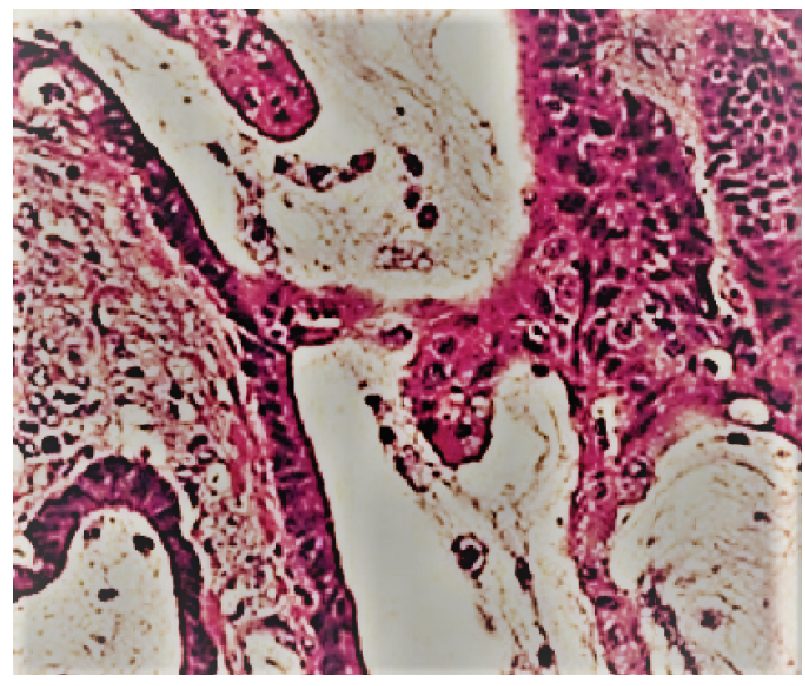

Figure 2. Histology showing mucinous adenocarcinoma.

of $E$. faecalis bacteremia is urogenital tract, but in our case the patient had no evidence of urinary tract infection, and no suspicion of acute cholecystitis and acute appendicitis

The patients with E. faecalis infective endocarditis most frequently have no identifiable source of bacteremia. The half of patients with $E$. faecalis infective endocarditis with unidentifiable source were found to have $\mathrm{CRC}$, when underwent colonoscopy in a study by Pericas et al [12]. In our case the colonoscopy was done because of the weight loss and anemia to look for malignancy, it was not intended to find the source of bacteremia.

Our patient was having AICD, and the presence of AICD and other implantable cardiac devices increase the risk of infective endocarditis. There is no evidence to suggest that the risk of E. faecalis endocarditis increases with having an implantable cardiac device. The previous population based studies have shown that staphylococci (S.aureus and S. epidermidis) were the most common bacteria cultured in patients with implantable cardiac devices and infective endocarditis [13].

The patient was also above age of 50, so needed colonoscopy for CRC screening anyways. But not all the cases of endocarditis with unidentified source can undergo colonoscopy, as some of these patients are sick and cannot withstand the fluid overload with valve regurgitation needed for colonoscopy preparation. So the generalization of colonoscopy for all the patients with $E$. faecalis endocarditis with unidentifiable source though seem reasonable may not be practical in some patients. Also, it seems E. faecalis infective endocarditis like S. bovis infective endocarditis has no impact on the prognosis and outcomes of CRC and most of the cases of CRC associated with infective endocarditis are at early stage. This goes with our case as well, which was diagnosed at T1N0M0 stage.

\section{Conclusions}

In conclusion, we here present a case of $E$. faecalis endocarditis with unidentifiable source with subsequent finding and as- 
sociation of stage $1 \mathrm{CRC}$ with the endocarditis, which was the source of $E$. faecalis bacteremia as well. We recommend that the patients with unidentified source of $E$. faecalis bacteremia and endocarditis regardless of age should undergo colonoscopy if feasible to identify underlying CRC at an early stage and timely treatment of it.

\section{Author Contributions}

Zubair Khan contributed in writing the case report and major parts of discussion. Nauman Siddiqui assisted in writing the manuscript. Wasif M. Saif supervised and reviewed the entire article.

\section{Disclosure}

I hereby confirm on behalf of all my co-authors and myself as well that we don't have any competing interest and we don't have any financial interest to disclose.

\section{References}

1. Alozie A, Koller K, Pose L, Raftis M, Steinhoff G, Westphal B, Lamprecht G, et al. Streptococcus bovis infectious endocarditis and occult gastrointestinal neoplasia: experience with 25 consecutive patients treated surgically. Gut Pathog. 2015;7:27.

2. Corredoira J, Grau I, Garcia-Rodriguez JF, Alonso-Garcia P, Garcia-Pais MJ, Rabunal R, Garcia-Garrote F, et al. The clinical epidemiology and malignancies associated with Streptococcus bovis biotypes in 506 cases of bloodstream infections. J Infect. 2015;71(3):317-325.

3. Baddour LM, Wilson WR, Bayer AS, Fowler VG, Jr., Tleyjeh IM, Rybak MJ, Barsic B, et al. Infective endocarditis in adults: diagnosis, antimicrobial therapy, and management of complications: a scientific statement for healthcare professionals from the American Heart Association. Circulation. 2015;132(15):1435-1486.

4. Habib G, Lancellotti P, Antunes MJ, Bongiorni MG, Ca- salta JP, Del Zotti F, Dulgheru R, et al. 2015 ESC Guidelines for the management of infective endocarditis: The Task Force for the Management of Infective Endocarditis of the European Society of Cardiology (ESC). Endorsed by: European Association for Cardio-Thoracic Surgery (EACTS), the European Association of Nuclear Medicine (EANM). Eur Heart J. 2015;36(44):3075-3128.

5. Scudeller L, Badano L, Crapis M, Pagotto A, Viale P. Population-based surveillance of infectious endocarditis in an Italian region. Arch Intern Med. 2009;169(18):17201723.

6. Ruoff KL, de la Maza L, Murtagh MJ, Spargo JD, Ferraro MJ. Species identities of enterococci isolated from clinical specimens. J Clin Microbiol. 1990;28(3):435-437.

7. Kajihara T, Nakamura S, Iwanaga N, Oshima K, Takazono T, Miyazaki T, Izumikawa K, et al. Clinical characteristics and risk factors of enterococcal infections in Nagasaki, Japan: a retrospective study. BMC Infect Dis. 2015;15:426.

8. Milbrandt E. A novel source of enterococcal endocarditis. Clin Cardiol. 1998;21(2):123-126.

9. Murray BE. The life and times of the Enterococcus. Clin Microbiol Rev. 1990;3(1):46-65.

10. Klein RS, Recco RA, Catalano MT, Edberg SC, Casey JI, Steigbigel NH. Association of Streptococcus bovis with carcinoma of the colon. N Engl J Med. 1977;297(15):800802.

11. Wang X, Allen TD, May RJ, Lightfoot S, Houchen CW, Huycke MM. Enterococcus faecalis induces aneuploidy and tetraploidy in colonic epithelial cells through a bystander effect. Cancer Res. 2008;68(23):9909-9917.

12. Pericas JM, Corredoira J, Moreno A, Garcia-Pais MJ, Falces C, Rabunal R, Mestres CA, et al. Relationship between enterococcus faecalis infective endocarditis and colorectal neoplasm: preliminary results from a cohort of 154 patients. Rev Esp Cardiol (Engl Ed). 2017;70(6):451458.

13. Carrasco F, Anguita M, Ruiz M, Castillo JC, Delgado M, Mesa D, Romo E, et al. Clinical features and changes in epidemiology of infective endocarditis on pacemaker devices over a 27-year period (1987-2013). Europace. 2016;18(6):836-841. 